\title{
Characterization of power quality transient phenomena of DC railway traction supply
}

\author{
Andrea Mariscotti \\ Università di Genova, Via Opera Pia 11A, 16145 Genova, Italy
}

DC railways are considered as an example of a complex distribution network, featuring distributed supply and moving loads. The Power Quality phenomena affecting traction voltage are many, steady and transient, depending on the converter operations at substations and on-board vehicles. A classification is proposed and a discussion follows of the correct methods for their quantification, with reference to related standards and to the vast background related to ac networks. The characterization of PQ is related on one side to ripple and fluctuation of the pantograph variables for supply purposes, and on the other side to the appearance of specific components relevant for interference to signaling on the track. Considerations and conclusions are based on a large amount of measured waveforms, recorded on the Italian railway networks, and are aimed at contributing to the forthcoming standards on traction supply quality.

Keywords: conducted interference; guideway transportation systems; power quality; spectral analysis; supply transients; time domain analysis

Citation: Andrea Mariscotti, Characterization of power quality transient phenomena of DC railway traction supply, Acta IMEKO, vol. 1 , no. 1 , article 8 , July 2012, identifier: IMEKO-ACTA-01(2012)-01-08

Editor: Pedro Ramos, Instituto de Telecomunicações and Instituto Superior Técnico/Universidade Técnica de Lisboa, Portugal

Received January $4^{\text {th }}, 2012$; In final form May $4^{\text {th }}, 2012$; Published July 2012

Copyright: (C) 2012 IMEKO. This is an open-access article distributed under the terms of the Creative Commons Attribution 3.0 License, which permits unrestricted use, distribution, and reproduction in any medium, provided the original author and source are credited

Funding: Information not available

Corresponding author: Andrea Mariscotti, e-mail: andrea.mariscotti@unige.it

\section{INTRODUCTION}

Power Quality (PQ) in railways encompasses a variety of phenomena, steady and transient: harmonic components (characteristic of the substation rectifiers), other similar components (not harmonically related to the supply frequency on the feeding ac side of substations, but caused by on-board converters and drives) and low frequency voltage fluctuations (due to more or less periodic load cycles) are examples of the first kind; steep current absorptions (in the form of the inrush current following the pantograph rise) and line voltage variations (due for example to pantograph bounce), and complex changes of modulation patterns (due to various types of transients, sudden tractive efforts and braking, wheel slip and slide, etc.) are examples of the second kind.

PQ in railway traction systems is relevant for several reasons: line voltage variations have influence on the pantograph voltage perceived by the single rolling stock and its performance [2]; harmonic distortion in combination with traction line impedance can trigger line voltage instability [2]; uncommon components in the absorbed current spectrum may lead to interference to signalling circuits when flowing back to substation as return current through the rails. More generally the PQ compatibility of a rolling stock unit with a railway network is recognized as electrical interoperability, part of the overall interoperability, aiming at ensuring the safe and efficient circulation across different railway networks in different countries, such as those of the European Union.

PQ properties and definitions are reviewed in section 2 with reference to standards and distinguishing those phenomena that are peculiar of $\mathrm{dc}$ distribution. In section 3 these elements are revisited and alternative methods are considered for their calculation. In section 4 measured data are used to verify both the considered methods and the relevance of the PQ issues occurring on real railway networks.

\section{REVIEW OF POWER QUALITY PHENOMENA}

Definition and analysis of PQ phenomena in ac railways are easier because they may be derived from ac supply networks [3], notwithstanding the different supply frequency, the distribution scheme and the presence of moving loads. In ac railways the emissions from substations and rolling stock are all of the harmonic type with reference to the common supply frequency [4], featuring a stability that is slightly worse than that of country-wide power grids [5][6]. In dc railways there is no such a synchronizing element and the emissions are harmonically related to independent sources: the substation keeps supplied by the dedicated feeders derived from the country-wide power grid, while for the rolling stock the motor frequency and the 
switching frequency of $\mathrm{dc} / \mathrm{dc}$ converters and inverters are the reference. The time window and the frequency resolution for spectrum analysis are thus not immediately following [3].

For ac and dc railways, besides power quality issues related to harmonic disturbance and their composition [4], network resonance and traction line - rolling stock interaction [7]-[9], a very basic requirement that impacts on train performance and service efficiency is the voltage level "seen" by the train pantograph, called useful voltage. The useful voltage $U_{a v, u}$ is defined in the EN 50388 [2] for dc railway systems as the average value of the mean value of the pantograph voltage $V_{p}$ (i.e. dc component) over a well-defined geographical area of the national network and for one or several trains. Thus, there is a distinction between the $U_{a v y, u}($ zone), for the average operation made over all the circulating trains in a given zone, and $U_{a v, u}$ (train), for the average operation made for one train over a predetermined journey or its timetable. The measurement data collected in the past and that will be used for the following analysis were all recorded on a single train and thus allow the calculation of $U_{a v, u}$ (train) only.

In general, PQ concept may be extended to a series of phenomena, steady and transient, that may have relevance for the whole railway network: impact on, and possible interference, to signalling circuits is required to be evaluated by analyzing the pantograph current spectrum against a so-called limit mask [10][11]. The analysis parameters are more or less fixed by the different national procedures adopted by the various administrations, yet there is no agreement on how to distinguish transient events: they bring non-characteristic spectral components and in some cases they are simply "recognized" and excluded from the analysis [12]. The focus here is on the identification of these phenomena on long-term recordings, so that they can be properly treated (in some cases processed separately, or used for the specific analysis of transient interference or simply discarded).

At $\mathrm{dc}$ it is not possible to use the fundamental period to derive an indication of the correct time window, both for harmonics and for the computation of the mean value. The time window duration has however a strong influence on how different types of transients are included and weighted, and this could lead to some uncertainty. This aspect is considered later on in section 3.

So far transient phenomena have been generically considered; the classification proposed in [13] is reported for the discussion:

- (Type 1) unusual sudden tractive efforts with packet-like current absorptions may trigger oscillations in the onboard filter and thus in the pantograph current $I_{p}$, but with negligible effect on $V_{p}$, due to the low short circuit impedance of the network;

- (Type 2) change over to an adjacent supply section connected to a different substation, while passing under a supply gap (the equivalent for dc of a neutral section for ac systems) and producing a $V_{p}$ step change;

- (Type 3) pantograph bounces disconnect the sliding contact from the contact wire for a few ms, depending on several factors (speed, mechanical performance of the pantograph frame and dampers, catenary oscillations, ice); this produces a step change of absorbed current $I_{p}$ and a spike like change of $V_{p} ; V_{p}$ is namely determined by the free response of the on-board circuitry for the short time interval during which the pantograph is detached from the catenary; it is remembered also that current conduction still occurs through the plasma at the pantograph-contact wire interface and this represents an increase of the supply resistance, thus explaining the $V_{p}$ steep but limited increase;

- (Type 4) change of the operating conditions and of the spectrum of current emissions of onboard converters due to wheel slip, internal control rules, driving style and applied torque, etc.

The next section is dedicated to the calculation methods and their settings to identify and evaluate these phenomena in terms of amplitude, spectral distribution and frequency of occurrence.

\section{CALCULATION METHODS AND PARAMETERS}

Without a fundamental frequency for the definition of harmonics, all the narrowband components are relevant for the calculation of the supply distortion. The distortion is thus put in relationship to ripple, defined below and particularly appropriate for the evaluation of the traction supply voltage.

The attention is on the pantograph voltage, even if the considered methods may be applied to the pantograph current as well. The ripple index defined below is a measure of the perceived quality applicable to dc networks [13][14]. Some types of transients and their influence on the spectral properties are then considered. The subject reveals to be particularly interesting also for the relationship with the interference to signalling systems, in particular if the variable of interest becomes the pantograph current. In any case a distorted pantograph voltage reflects in a distorted pantograph current if the locomotive can be considered simply as a passive load at those frequencies.

\subsection{Ripple and useful voltage}

Ripple is defined as the variation of a quantity about its steady state value during steady electric system operation [3]. Ripple is interpreted often as a periodic variation around the steady state dc value, but not necessarily so [15][16]: some components are related to steady periodic sources (harmonics of rectifiers and inverters [17]-[19] in steady conditions), but others are caused by transients (interpreted as aperiodic phenomena of limited duration).

The expressions defined in [20] are briefly summarized. Given the $q(t)$ quantity (in this case the pantograph voltage $V_{p}$ ), the peak-to-peak value $q_{p p}$ over $m_{T}$ samples interval is the exact ripple index, RI, that is the maximum peak-to-peak value over a given time interval, and is given by

$$
q_{p p, T}=\max _{n, m}\left\{q[n]-q\left[n+m+m_{T}\right]\right\} .
$$

If a spectrum-based approach is followed, a sliding DFT over a time window $T$ is computed giving the spectrum $Q[k]$ [21][22].

$\mathrm{RI}$ is then calculated as the sum of the components of the set $K_{t b}$, containing values of the index $k$ with corresponding component $Q[k]$ with amplitude larger than a given threshold thr; Sum-of-Amplitudes (SA) and Sum-of-Amplitudes-andPhases (SAP) rules, as given in [20]

$$
q_{D F T, T, S A}=\sum_{k \in K_{t b r}}|Q[k]|
$$

and

$q_{D F T, T, S A P}=\left|\sum_{k \in K_{t b r}} Q[k]\right|$. 
The thr value must be carefully chosen not to leave out any significant component and to keep the size of $K_{t b r}$ as low as possible; normally a $0.1 \%$ relative to the nominal voltage is already a conservative choice, provided that full-scale is correctly set. Lower values may be used in the presence of many spectral components, but only in case of a background noise that is located well below. The purpose of thr is to "clean" the spectrum, ruling out all spectral components other than the relevant ones not to artificially magnify the resulting RI value. Holding these assumptions a lower thr value, such as $0.01 \%$ used in the next figures, represents only a more cautious choice, but does not change the result.

An overlap factor $p$ of 0.5 was shown to adequately track signal dynamics and is compatible in terms of minimum correlation for the large part of used windows. In [13] it is shown that the two indexes differ slightly and therefore only SA will be considered in the following.

$U_{a v, u}$ is the index that is evaluated to assess the adequacy of the infrastructure (power supply network) to the prescribed performance of the circulating rolling stock and concerns only the average value, while the proposed RI takes account in a similar way of the other spectrum components. For $3 \mathrm{kV} d c$ railways the minimum $U_{a v, u}$ is set to $2800 \mathrm{~V}$ for high speed lines and $2700 \mathrm{~V}$ for conventional lines.

\subsection{Periodic phenomena}

With similarity to harmonics in ac railways, periodic phenomena in dc railways are related to the commutation of static converters either at substations (6-pulse and 12-pulse diode rectifiers) or on-board (the front-end choppers have a switching frequency of some hundreds $\mathrm{Hz}$, possibly changing in discrete steps for variable operating conditions; the conducted emissions of traction drives may leak back to the dc line, appearing as sixth multiples of the motor stator frequency and/or higher components around the PWM switching frequency). Changes to the operating conditions, such as a change of speed and thus of mechanical rotational speed, may occur leading to a Type 4 transient, highly dependent on the type of locomotive and the adopted modulations and control schemes.

The requirements on frequency resolution $d f$ (and thus on the time window duration $T$ ) are set by several national standards for evaluation of interference to signalling, still following a DFT approach. In this case $d f$ is set to $1 \mathrm{~Hz}$ [12] or slightly larger and the average of successive spectra may be required; the spectrum is extended up to some $\mathrm{kHz}, 3600 \mathrm{~Hz}$ for the Italian case. The most modern approach envisaged by the recent CLC/TS 50238-2 [23] is that of using a bank of band-pass filters followed by an integral calculation of the rms value [24].

It is also underlined that a coarser frequency resolution allows fast tracking of transient components. Being the relevant components for interference located in the higher portion of the frequency axis, a $d f$ of e.g. $5 \mathrm{~Hz}$ leads to a few $\%$ frequency resolution and allows a $200 \mathrm{~ms}$ base time resolution, that can be further reduced by means of overlap.

\subsection{Aperiodic transients}

The complex scenario of variable operating conditions, position of the rolling stock and the possibility of local unstable conditions of traction converters located on different nearby vehicles, give rise to a wide set of transients. As already pointed out in the classification given in section 2 , transients of the pantograph voltage and/or current may occur due to the inrush current of the vehicle filter, pantograph bounces, wheel slipping and sliding, etc. The aim is twofold: identifying transients during the measurements of conducted emissions on the pantograph current (subject to the values of the analysis parameters set by the standards for interference to track circuits) and during the evaluation of the useful voltage (with much less constraints on the parameters settings). Broadly speaking the former may require a finer frequency resolution to match with the bandwidth and operating frequency range of the victim track circuits and related limit prescriptions. The latter is mostly influenced by slow variations, such as those experienced while moving at the end of a supply section, farthest from the substation (as it will be commented for Figure 1 at points (1) and (2)); the analysis can be made with a less demanding frequency resolution and thus a finer time resolution.

A $10 \mathrm{~Hz}$ frequency resolution will be normally used in the following, accompanied by a $50 \%$ (or $75 \%$ ) overlap.

\section{REAL CASES}

Recordings taken on the Italian $3 \mathrm{kV}$ dc railway lines are considered to evaluate the RI, to identify the transients, their spectral characteristics and their influence on the $U_{a v, u}$ and in general on the spectrum components. The following subsections are aimed at evaluating the spectral properties of transients and defining the best indexes to identify and isolate the transients themselves.

\subsection{Useful voltage and ripple index}

The useful voltage has been computed from the measured pantograph voltage $V_{p}$ using a $1 \mathrm{~s}$ average interval (as prescribed by the EN 50163 [5] and EN 50388 [2]) for various test runs performed on different Italian lines (details are given in the caption of Figure 1).

It is underlined that the $U_{a l, u}$ computation here includes all the operating conditions of the train rather than only traction, thus explaining the high overvoltages on the Chiusi-ArezzoOrte line, probably occurring during regenerative braking in particular line conditions). The processed $V_{p}$ recordings give only in one case a useful voltage lower than the prescribed limits [2], $2800 \mathrm{~V}$ and $2700 \mathrm{~V}$, for high speed and conventional interoperable dc lines. In the Arezzo-Chiusi-Orte recordings some overvoltages are present, with a value much higher than the indicated $U_{\max 3}$, the maximum non-permanent voltage as per EN 50163. The observed overvoltages last for one or two average samples, so they have a time duration nearly equal to or lower than $1 \mathrm{~s}$ and thus fall under the category of transient overvoltages.

A 10 min profile of $V_{p}$ and $I_{p}$ is shown in Figure 2, together with the SA index, that differs from the one computed in [13] for the lower threshold $\operatorname{thr}(0.01 \%$, rather than $0.1 \%)$ and the use of $75 \%$ overlap. This recording shows quite a regular profile of current absorption and the large step changes in $V_{p}$ are due to supply gaps (Type 1 transient). The $V_{p}$ box-like increase (3) is probably due to a particular arrangement approaching a station, confirmed by the decrease of $I_{p}$ at the end of the recording.

A fairly constant high value $I_{p}$ profile represents medium/high speed travelling on straight lines with rare stops, such as on main lines. Even in this case pantograph bounces, wheel slip/slide and transitions in the operating conditions can produce transients with effects visible in the voltage and current spectra. 


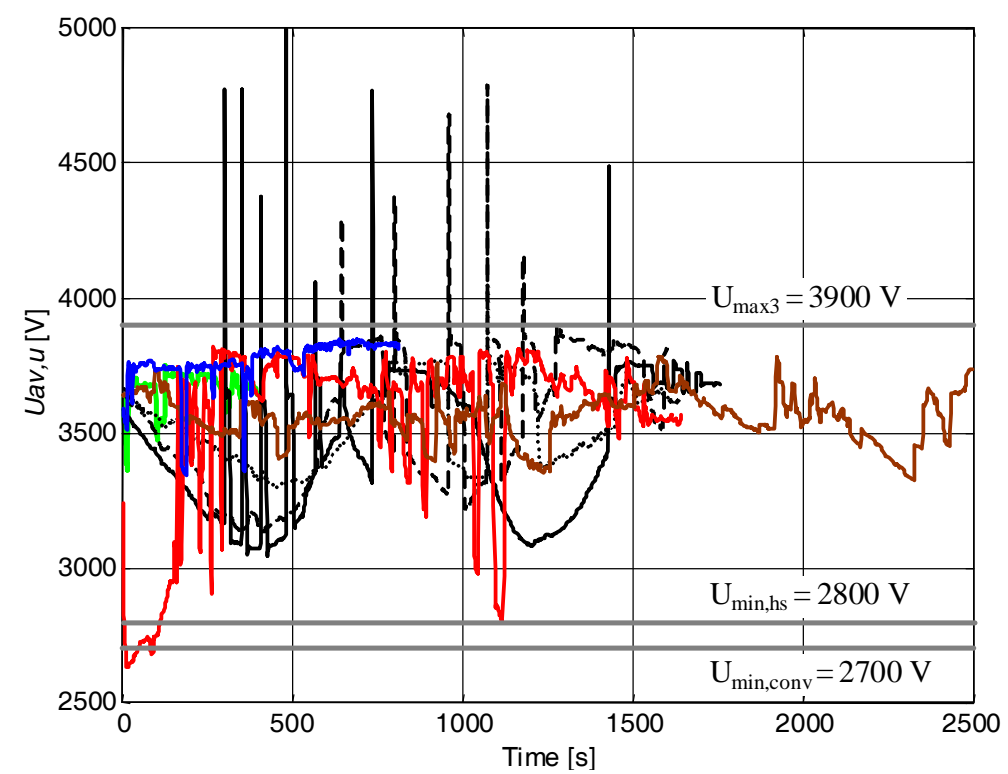

Figure 1. $U_{a v, u}$ for runs of different duration: Arezzo-Chiusi-Orte (black), Torino-Modane (blue), Gemona-Tarvisio (green), Genova-Ventimiglia (red/brown).

Figure 2 is referred to a heavy loaded line, with the absorbed current in the range of 1700-1800 A, corresponding to an absorbed power of about 5.8 to $6.3 \mathrm{MW}$. The corresponding voltage ripple is however limited to about $2 \%$, occurring only at some time intervals. At point (4) RI has a step-like reduction, not only due to the increase in $V_{p}$ dc value, but also due to lower supply impedance.

Figure 3 (with the same overlap and thr values of Figure 2) shows an interval of lightly loaded operations with the RI that is nearly half that of Figure 1, as the reduced absorbed current implies. Moreover the pantograph voltage is at the maximum values compatible with the $3 \mathrm{kV}$ dc system [5] (the test run began close to a supply substation of a normally highly loaded line, so regulated for the maximum allowed no-load voltage); in this case the RI normalized by its value is further reduced. It is interesting to note that in the first $100 \mathrm{~s}$, characterized by a negligible RI, time-varying harmonics are populating the spectrum (see section 4.2).

\subsection{Spectral components}

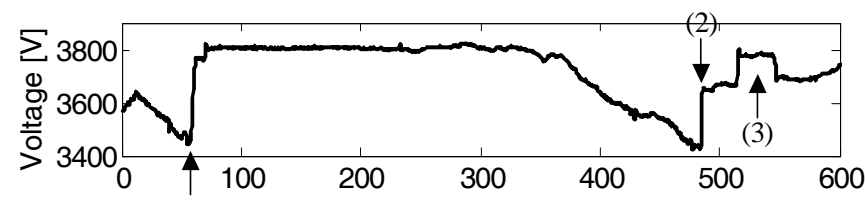

(1)
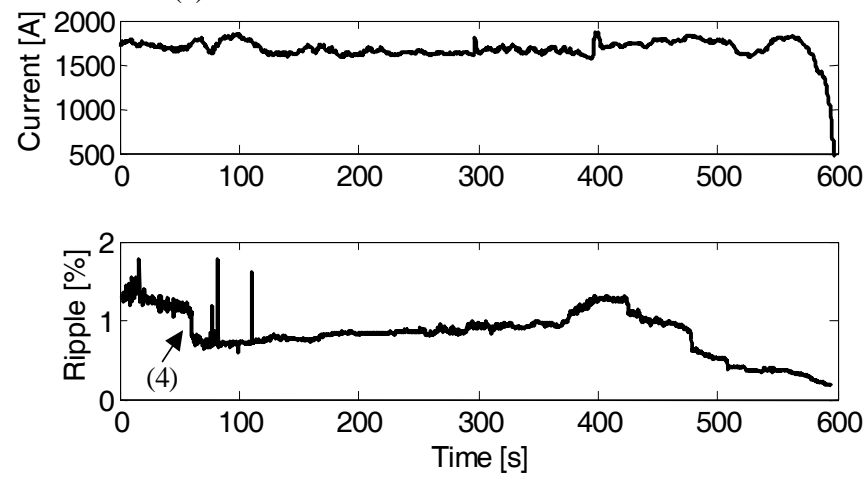

Figure 2. Ripple Index: SA curve for a 10 min test run.
When major transients are not visible in the time domain waveforms, this does not imply that other types of transients may be present. These transients are mainly of Type 1 (bounded to the low frequency range) or Type 4 (occurring over the entire frequency interval).

Figure 5 through 6 show the spectra at low frequency and around one of the PWM bands of the traction drive for an apparently almost constant RI interval (corresponding to $t^{*}=5.8 \mathrm{~s}$ in Figure 2). Low frequency broadband components due to leakage often cover important changes to high frequency components of smaller amplitude. Transients of this kind produce several components that may be the reason for noncompliance with signalling interference limits [23] during dedicated tests and must be correctly weighted in the overall operating profile of the converter under test [25]. Leakage at very low frequency occurs because of the non-stationary nature of supply voltage fluctuations. The best tool to identify and to track spectrum occupation is the time-frequency spectrogram.

Figure 5 reports the spectrum of the pantograph current, to
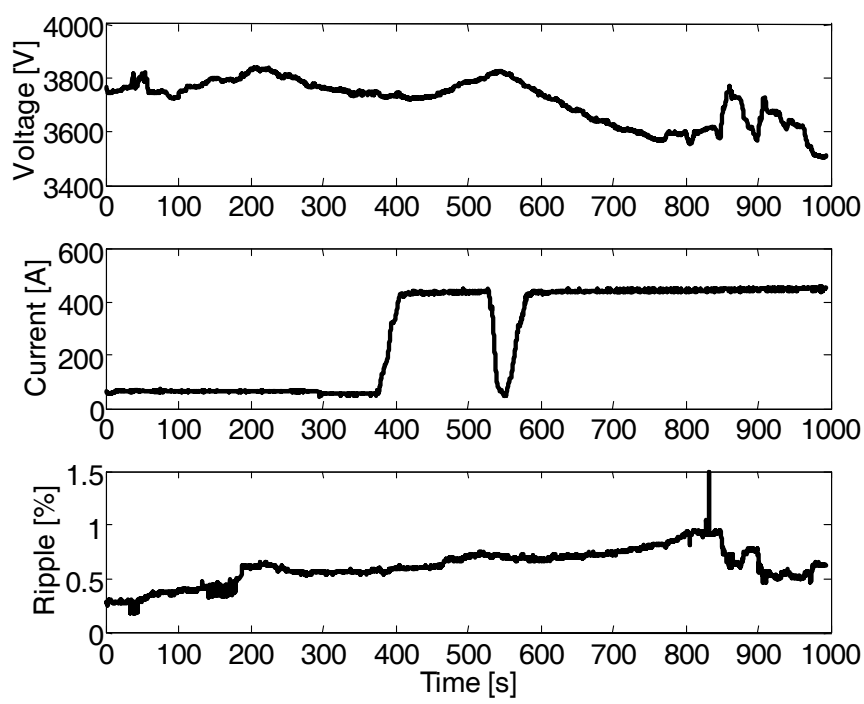

Figure 3. Ripple Index: SA curve for a 17 min test run. 
identify the spectrum components more easily; the components of the pantograph voltage spectrum are immediately related to them by the line impedance seen at the pantograph. In Figure 5a two components at 150 and $490 \mathrm{~Hz}$ definitely increase after $t^{*}$, besides an evident frequency leakage below $80 \mathrm{~Hz}$; zero stuffing is used in Figure $5 \mathrm{a}$ to enhance the frequency resolution down to $5 \mathrm{~Hz}$, identifying the peculiar behaviour below $80 \mathrm{~Hz}$ probably due to the resonant free response of the on-board filter, but surely influenced by the frequency leakage of the dc component. For the aim of reducing the latter it is advisable to subtract an estimate of the dc component, bringing the analysed signal down to zero mean. In Figure $5 b$ at higher frequency the dotted and solid black spectra at or after $t^{*}$ prevail, showing an increase also of high frequency components. Two components are almost fix, $300 \mathrm{~Hz}$ (substation) and $560 \mathrm{~Hz}$ (on-board chopper).

The raw Fourier spectra need to be pre-processed before being used for the RI calculation and other computations, since the background noise components may largely influence an overall index like RI or the total rms; a threshold needs to be assigned to zero out the components below it. When evaluating broad peaks with a fine frequency resolution, peak isolation is necessary to avoid counting them more than once, including lateral components due to leakage. These techniques have already been successfully applied to the processing of recordings of electromagnetic field intensity on-board rolling stock [26]. However the use of a threshold, overlap percentage and peak isolation is prone to large deviations, as it is evident, by comparing the results in Figure 2 with the preliminary results reported in [13]. It is however recognized that the RI index is a useful tool to locate various types of transients on the time-axis for further processing and analysis.

From a graphical point of view, the time-frequency representation of Fourier spectra is an effective tool. Transients and time-varying components leave a clear signature in the Fourier spectra computed by Short Time Fourier Transform, or spectrogram. As it is known, the spectrogram is subject to the same constraint on the frequency and time resolution of the basic Fourier analysis.

The steady characteristic harmonics at $300 \mathrm{~Hz}, 600 \mathrm{~Hz}$ and $900 \mathrm{~Hz}$ may be easily seen in Figure 4(a); other steady noncharacteristic harmonics at $50 \mathrm{~Hz}, 100 \mathrm{~Hz}$ and $150 \mathrm{~Hz}$ are located in the lower part of the frequency range. A comb pattern of harmonics referred to a fundamental around $280 \mathrm{~Hz}$ is also present $(280 \mathrm{~Hz}, 560 \mathrm{~Hz}, 840 \mathrm{~Hz}, 1120 \mathrm{~Hz})$, that increases with increasing speed and reaches approximately $460 \mathrm{~Hz}, 940 \mathrm{~Hz}, 1400 \mathrm{~Hz}, 1860 \mathrm{~Hz}$ after $30 \mathrm{~s}$; the estimated rate of rise is thus $6 \mathrm{~Hz} / \mathrm{s}$. This is evidently the emission of a traction static converter during acceleration and represents a typical Type 4 transient.
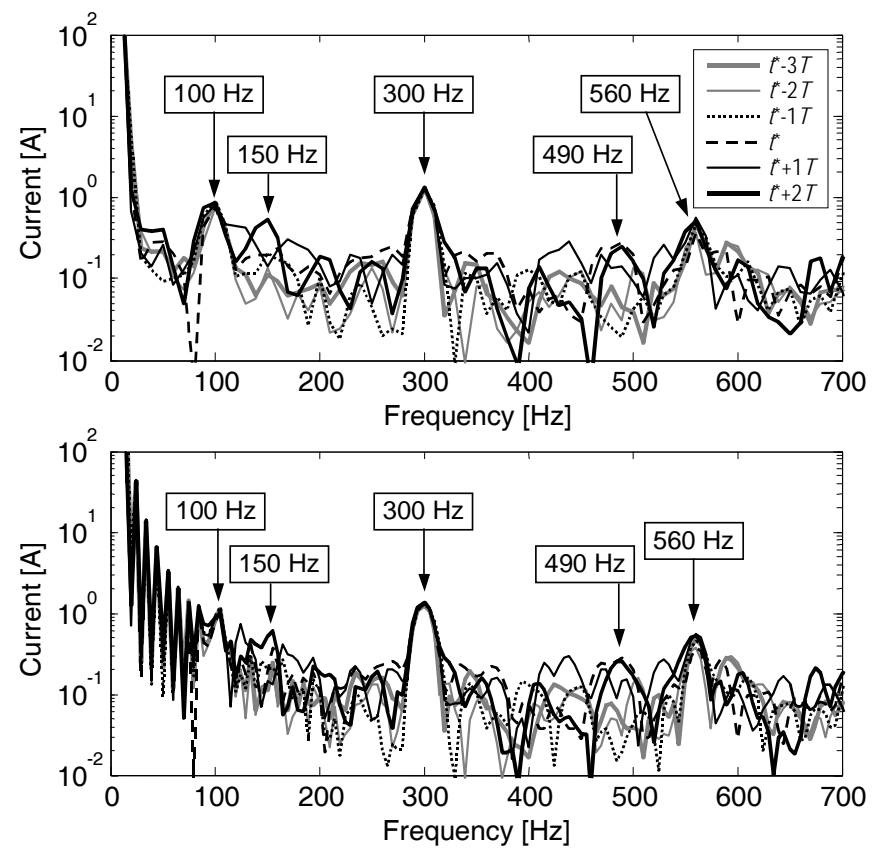

(a)

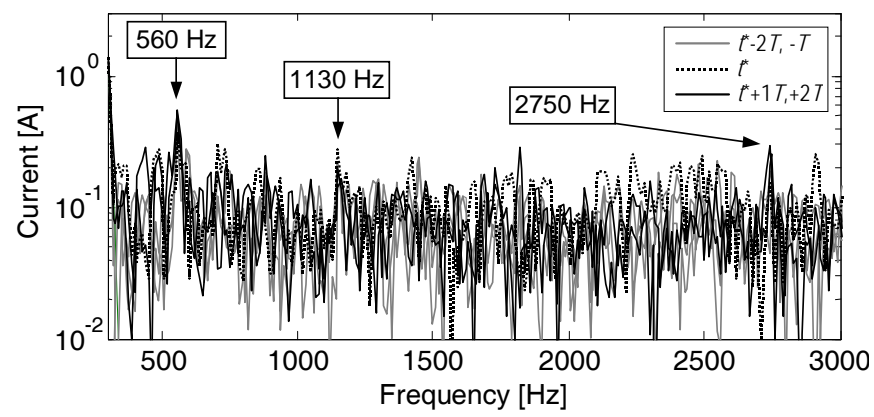

(b)

Figure 5. Analysis of transient behaviour at $t^{*}=5.8 \mathrm{~s}$ : (a) low frequency, with base and enhanced frequency resolution; (b) high frequency.

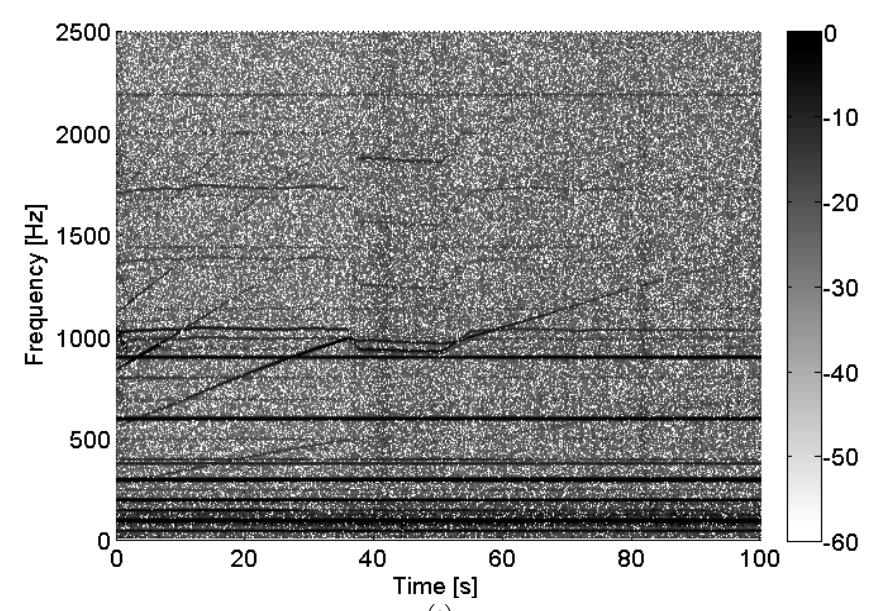

(a)

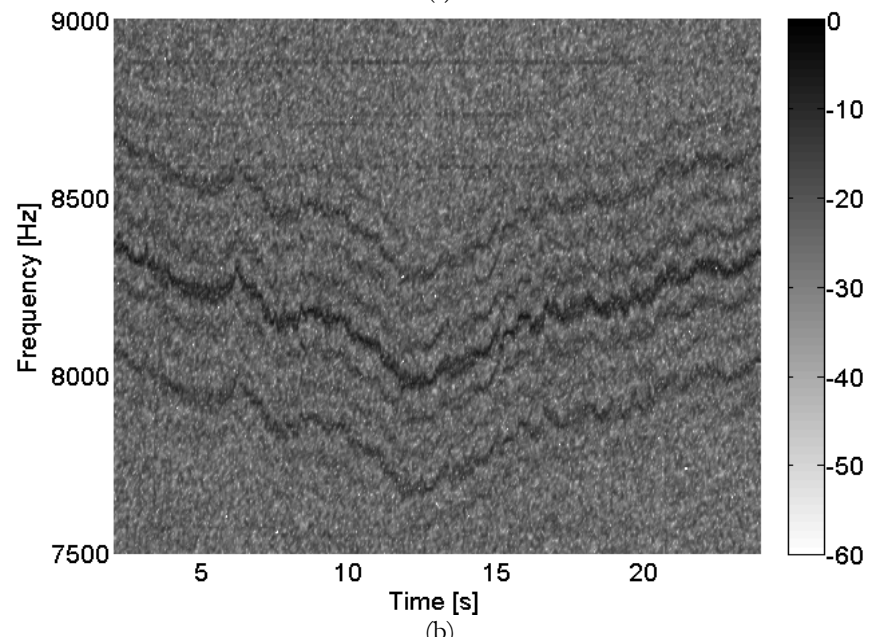

(b)

Figure 4. Spectrogram in dBV: (a) low frequency harmonics are artificially compressed to highlight time-varying harmonics; (b) typical non-stationary PWM signature from a different test run. 
The spectrogram in Figure 4a also shows two vertical lines at 40 and $80 \mathrm{~s}$, corresponding to transients of Type 2 or 3 . In Figure $4 \mathrm{~b}$ the high frequency pattern of the PWM modulation of a variable speed drive, probably the traction drive, is also clearly visible. The components are located symmetrically around the higher central component. The evolution versus time follows the operating conditions of the rolling stock, i.e. speed and tractive effort. This type of emissions, also of Type 4, is particularly relevant when interference to signalling is concerned. Limits for track circuits are expressed with a bandpass approach and non-stationary components may fall inside the susceptibility band [10][23][24].

The spectrogram is particularly useful in detecting such transients if a trade-off between frequency and time axis resolution is reached. Post-processing of the amplitude and graphical presentation can ease visual detection.

Two transients have been selected and further analysed in Figure 6 and Figure 7 with a frequency resolution of $25 \mathrm{~Hz}$ (40 $\mathrm{ms}$ time window) and overlap of $50 \%$; the overlap is necessary not only to track time varying components, but also to artificially increase the time axis resolution.

The periodicity of about $200 \mathrm{~Hz}$, visible in Figure 8, is related to the two peaks at about $5-6 \mathrm{~ms}$ in Figure 7 ; on the contrary the single peak of Figure 6 produces a flat spectrum. In both cases the transient components mask the characteristic harmonics, such as $300 \mathrm{~Hz}$.

The last plot, in Figure 8 is obtained with a $90 \%$ overlap, so that the time step is only $4 \mathrm{~ms}$; it is possible to distinguish the spectra before and after the transient event (dashed and dotted black curves), the spectra just at the beginning and the end of it (solid black curves) and during the transient itself (grey curves).

Overlapping and zero stuffing can improve the time axis resolution, ensuring transient detection; based on normal transient durations ( 2 to $10 \mathrm{~ms}$ ), a $25 \mathrm{~Hz}$ resolution with $50 \%$
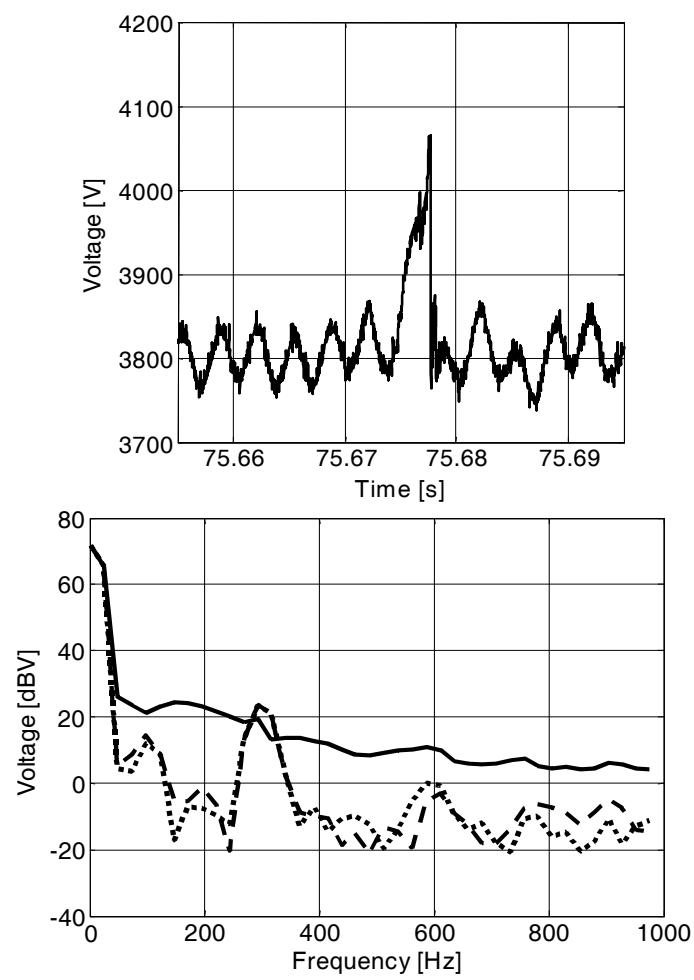

Figure 6. Spectrogram: transient waveform and Fourier spectra before (dashed), at (solid) and after (dotted) the transient (20 ms time step).
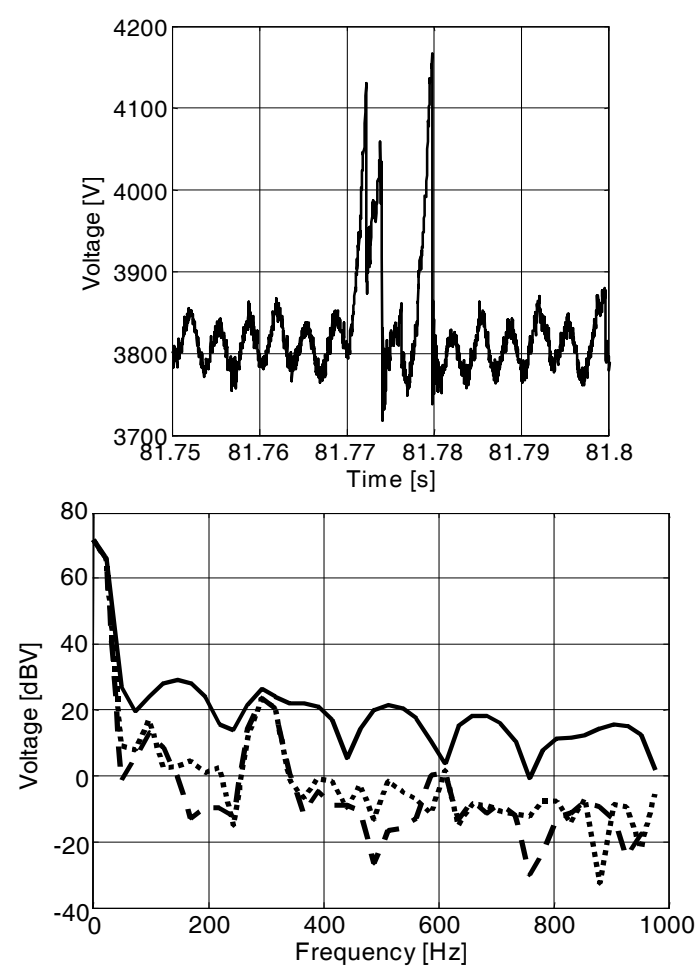

Figure 7. Spectrogram: transient waveform and Fourier spectra before (dashed), at (solid) and after (dotted) the transient (20 ms time step).

overlap ensures a $20 \mathrm{~ms}$ time step and a uniform increase of spectrum components by about $30 \%$. Smaller time steps can be used simply increasing the overlap to $75 \%(10 \mathrm{~ms})$ or $90 \%$ ( $4 \mathrm{~ms})$. With the latter time step and the base $25 \mathrm{~Hz}$ resolution, also the single pantograph voltage spike can be detected and correctly processed with acceptable "dilution" over the $40 \mathrm{~ms}$ window.

The analysis is repeated for another transient with a finer frequency resolution and a better time axis representation (Figure 9). The $300 \mathrm{~Hz}$ component is visible as the main ripple term both in time and frequency domains; the grey circles identify a three-by-three pattern due to the $100 \mathrm{~Hz}$ component. The transient occurring at $15.25 \mathrm{~ms}$ produces the broad spectrum shown in heavy black, but the extinction of the transient in about $1 \mathrm{~ms}$ is followed by the free response of the on-board filter, that lasts for about $10 \mathrm{~ms}$, modifying the regular pattern of $300 \mathrm{~Hz}$ and $100 \mathrm{~Hz}$ components (see the two grey arrows on the following two $300 \mathrm{~Hz}$ cycles) and produces the

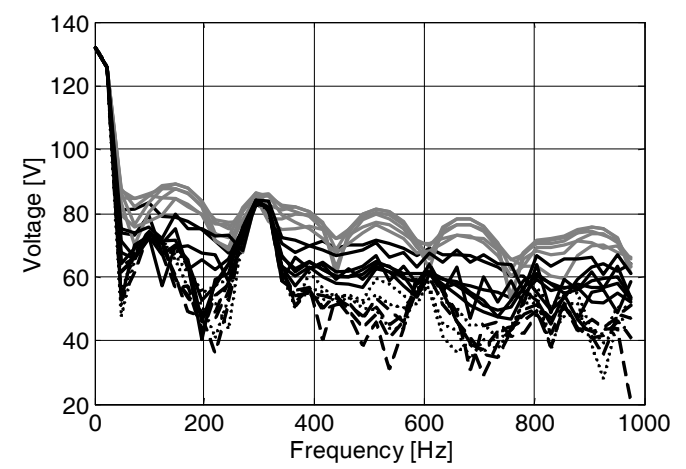

Figure 8. Spectrogram (same as Figure 7): spectra before (dashed black), partially including (solid black), centred at (solid grey) and after (dotted black) the transient (4 ms time step). 
transient spectrum components indicated by the black arrows. The spectrum components during the transient are predominant up to about $500 \mathrm{~Hz}$, with an amplitude increase of approximately $10 \mathrm{~dB}$.

Another uncommon type of transient is related to the stability of the supply frequency which, for dc traction systems supplied by the national grids, is $50 \mathrm{~Hz}$ in Europe and $60 \mathrm{~Hz}$ in a few other countries (e.g. Japan, Korea, United States, etc.). The analysis of the Arezzo-Chiusi-Orte recordings looking for Type 2 or Type 3 transients revealed a temporary shift of substation characteristic harmonics due to a supply frequency change, as shown in Figure 10.

In Figure $10 \mathrm{a}$ the $V_{p}$ waveform and the RI curve are shown only for reference.

The spectrogram in Figure 10b using a logarithmic frequency scale reveals repeated low frequency transients of moderate amplitude, indicated by the repeated dark and clear vertical lines, extending up to about the $50 \mathrm{~Hz}$ component. The most relevant transients at $101.0 \mathrm{~s}$ and $101.4 \mathrm{~s}$ extend above it, up to the $100 \mathrm{~Hz}$ component.

The linear scale in Figure 10c reveals a frequency shift of all substation characteristic harmonics that can be led back to a significant change of the $50 \mathrm{~Hz}$ supply frequency of nearly $0.7 \mathrm{~Hz}$ (estimated from the higher order harmonics, where the frequency shift is proportionally larger and more easily evaluated). This event is quite uncommon for the observed relevance and the effect is a shift of various substation characteristic harmonics that might fall inside the operating frequency intervals of signalling devices. Frequency stability is not further considered here; a complete discussion of this topic for ac traction supplies can be found in [6][28], where the variations of the fundamental are however much smaller with a span in the order of $0.1 \mathrm{~Hz}$.

\subsection{Wavelet analysis}

The considerations on the classical Fourier analysis of section 4.2, with the frequency resolution chosen with
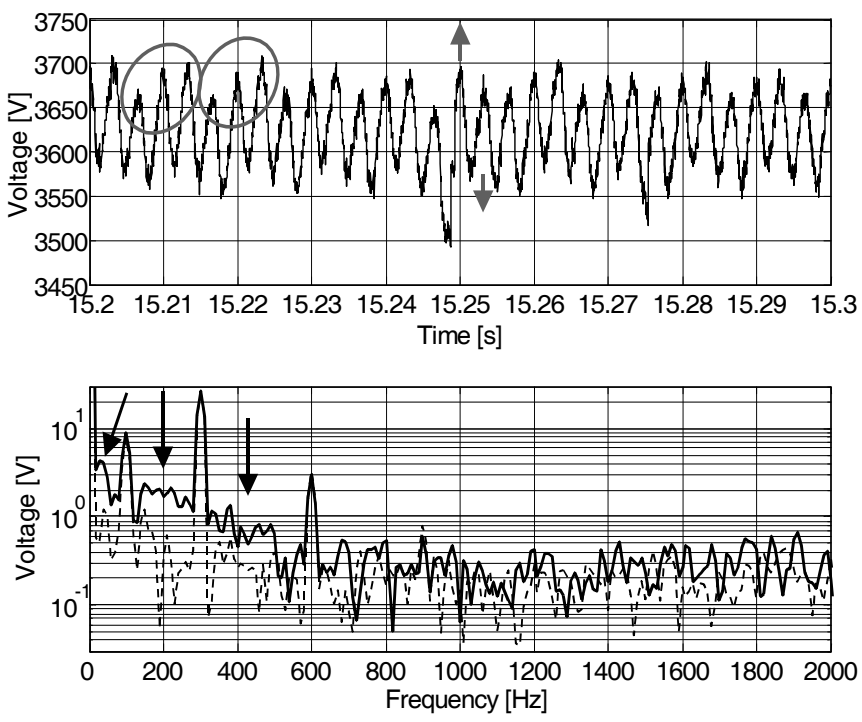

Figure 9. Spectrogram: transient waveform and Fourier spectra before (dashed) and at (solid) the transient (10 ms time step, $50 \%$ overlap); the arrows in the time domain trace identify a change in the regular pattern of the $100 \mathrm{~Hz}$ and $300 \mathrm{~Hz}$ components, the three arrows in the frequency domain spectrum identify the frequency intervals where the effect of the transient is evident. constraints on the time resolution, suggest the use of a wavelet representation for transients of Type 1,2 and 3.

The Discrete Time Wavelet Transform (DTWT) is at the moment preferred for its simplicity with respect to the Continuous Wavelet Transform, notwithstanding the better performances of the latter in terms of time and frequency resolution [27]. Transients in $V_{p}$ are detected, by applying a threshold to the details $d_{k}$, and classified, by deriving an
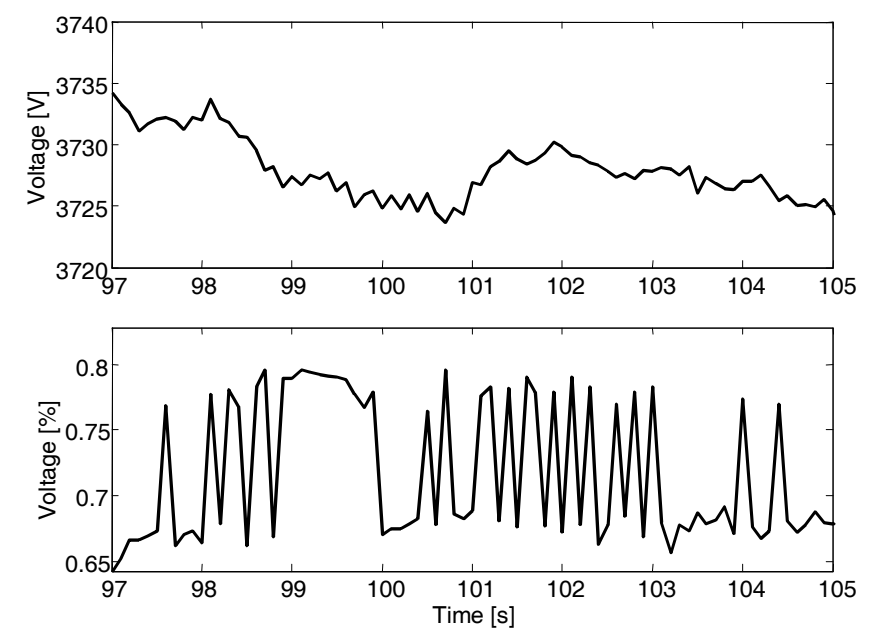

a)

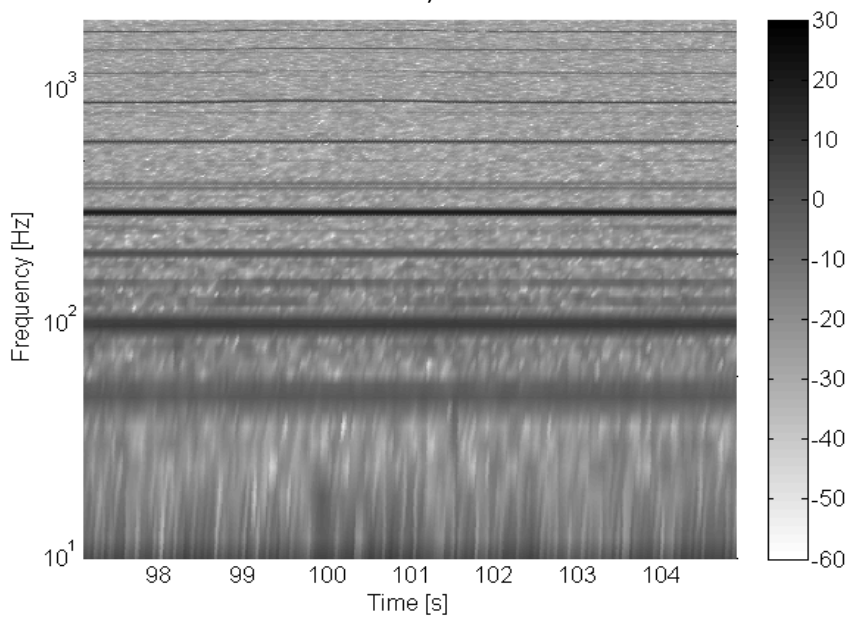

b)

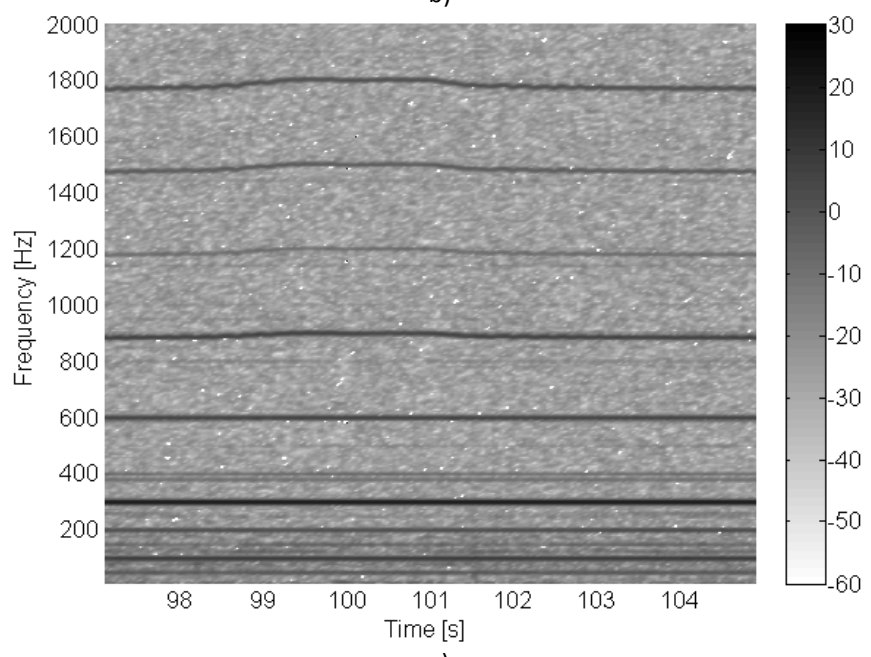

c)

Figure 10. In a) the pantograph voltage, $V_{p}$ (top) and the Ripple Index, RI (bottom). In b) and c) the spectrogram in dBV (10 Hz frequency resolution, $75 \%$ overlap) in logarithmic and linear frequency scale respectively. 
empirical characterization of amplitude and oscillations in each detail. Figure 11 shows an example of a Type 3 transient at $70.29 \mathrm{~s}$; a pantograph bounce is superimposed on the steady $V_{p}$ ripple of substation harmonics (an interpretation of the main ripple components visible in the time domain waveform was done after Figure 9).

The details $\mathrm{d} 3$ and $\mathrm{d} 4$ show the $300 \mathrm{~Hz}$ ripple, that is absent from the adjacent details. Wavelet details can be interpreted as band-pass channels with a centre frequency that, for DTWT, is determined by a geometric ratio. The basic frequency $f_{b}$ is determined by the sampling frequency and by the type of the wavelet; the centre frequency values at each scale $a$ (and detail) are readily determined as $f_{b} / 2^{a}$. Details not interested by the substation ripple are the candidates for the transient detection (see Table 1). The best mother wavelet is then selected by considering the correct band apportioning, the amplitude of the peaks appearing in the details and the accuracy of their time location. Many mother wavelets produce almost identical results [13]; the comparison is carried out by analysing the frequency behaviour of the examined wavelets. In the following "db" will stand for Daubechies and "sym" for Symlet.

The values reported in Table 1 are referred to a sampling time of $160 \mu \mathrm{s}$, eight times slower than the original sampling time. To cover a wider frequency interval, a lower value is needed and a larger number of details, thus increasing the computational effort.

It is remembered that pantograph bounces trigger the free response of the on-board filter, located in the lower portion of the frequency axis (see Figure 9), and modify temporarily the conducted emissions of the on-board converters, so that they represent a relevant event for the evaluation of interference to signalling circuits, in particular in the low and medium frequency intervals.

In [28] the $d b 4 / d b 6$ and $d b 8 / d b 10$ were found the best choices for fast and slow transients, but for ac industrial supply networks. Two wavelets ( $d b 3$ and $d b 5)$ are tested first and the results are shown in Figure 12 and Figure 13.

Low order wavelets may be not suitable to track all the variations of the analysed signal, but they feature a slightly larger peak amplitude in the low order details and a nonoscillating behaviour, thus ensuring a better time axis location of the crossing of the chosen threshold value and a nonambiguous behaviour (see Figure 14 for a $d b 1$ wavelet applied to the same signal trace). Without showing the results in the caption of Figure 14 it is stated that the results are practically identical to those obtained with "sym1" and "Haar" wavelets. Given the use of discrete values to define the latter, the same is highly indicated to reduce the required computational effort and implement a real-time function.

Setting the sampling frequency to a higher value $(25 \mathrm{kHz}$,

Table 1. Frequency axis location of wavelets.

\begin{tabular}{cccc}
\hline & \multicolumn{3}{c}{ Wavelet type } \\
& $\boldsymbol{d b 1}$ & $\boldsymbol{d b 3}$ & $\boldsymbol{d b 5}$ \\
\hline $\boldsymbol{d t}[\boldsymbol{b s}]$ & 160 & 160 & 160 \\
$\boldsymbol{f}_{c 1}[\mathrm{~Hz}]$ & 3113 & 2500 & 2083 \\
$\boldsymbol{f}_{c 2}[\mathrm{~Hz}]$ & 1556 & 1250 & 1042 \\
$\boldsymbol{f}_{c 3}[\mathrm{~Hz}]$ & 778.2 & 625 & 520.7 \\
$f_{c 4}[\mathrm{~Hz}]$ & 389.1 & 312.5 & 260.4 \\
$f_{c 5}[\mathrm{~Hz}]$ & 194.6 & 156.3 & 130.2 \\
\hline
\end{tabular}
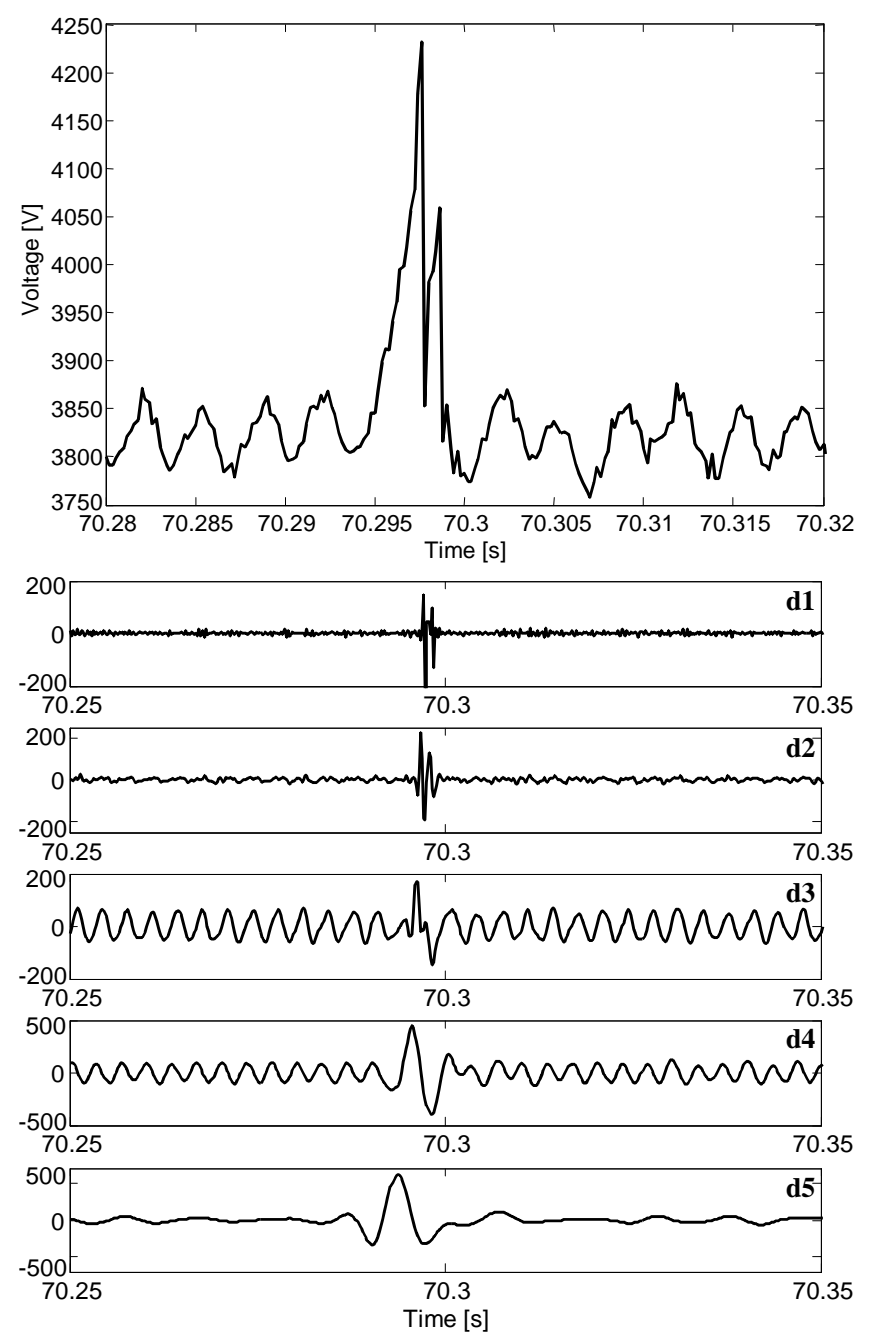

Figure 11. $V_{p}$ transient analysed with db3, 5 levels.

four times larger than the previous one used to derive Table 1 and Figure 11, Figure 12 and Figure 13), transients with a steep rise time and a time duration limited to one or few milliseconds may be detected more effectively (as shown in Figure 15, that corresponds to Figure 13 in [13]). Such a sampling time moves all the five details on a band above the main characteristic harmonics, being $f_{5}$ located at $778 \mathrm{~Hz}$.

\section{CONCLUSIONS}

In the present paper a range of transients typical of $\mathrm{dc}$ railway systems are considered and classified for their time and frequency behaviour. The target of the analysis is the evaluation of the power quality perceived at the pantograph voltage, but also the identification and location on the time axis of transients, that are relevant for interference to signalling circuits and thus for interoperability. The use of spectrogram and wavelets is proposed for the location of pantograph bounces on long records, that amount to many Gigabytes of data and cannot be inspected manually; wavelets types and the behaviour of the details were tested based on real signals. The results available in the literature, that advise the optimal settings for wavelet analysis, are almost always referred to ac distribution networks in industrial systems, while a dc railway system represent a peculiar case study. Very simple mother wavelets, such as a Daubechies or Symlet of order 1 or the Haar wavelet, seem preferable (in particular the latter), if accurate time axis location and fast computing are the main requisites. 


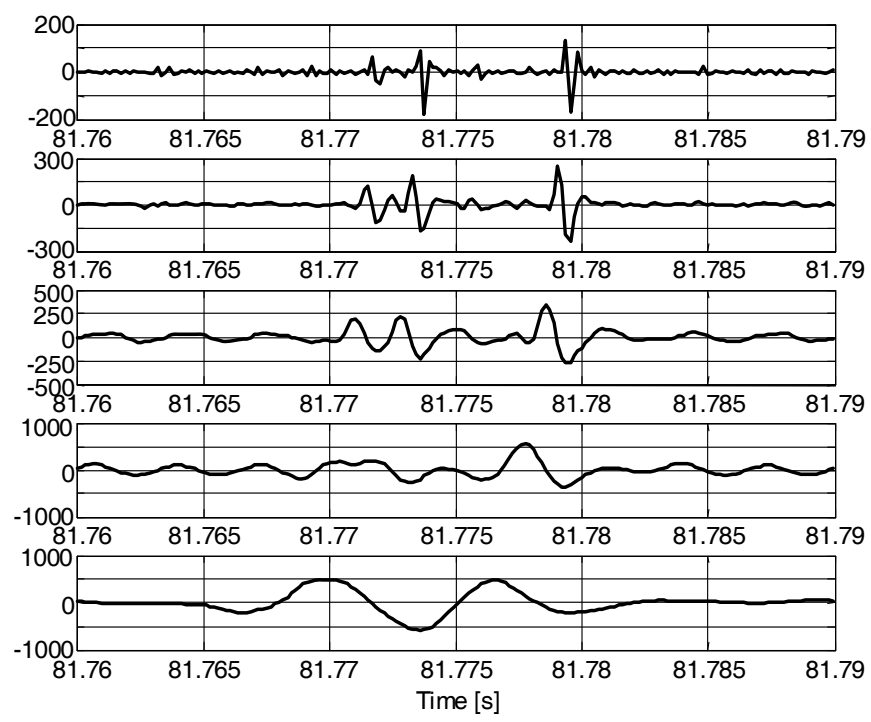

Figure 12. $V_{p}$ transient of Figure 8: db3, 5 levels.

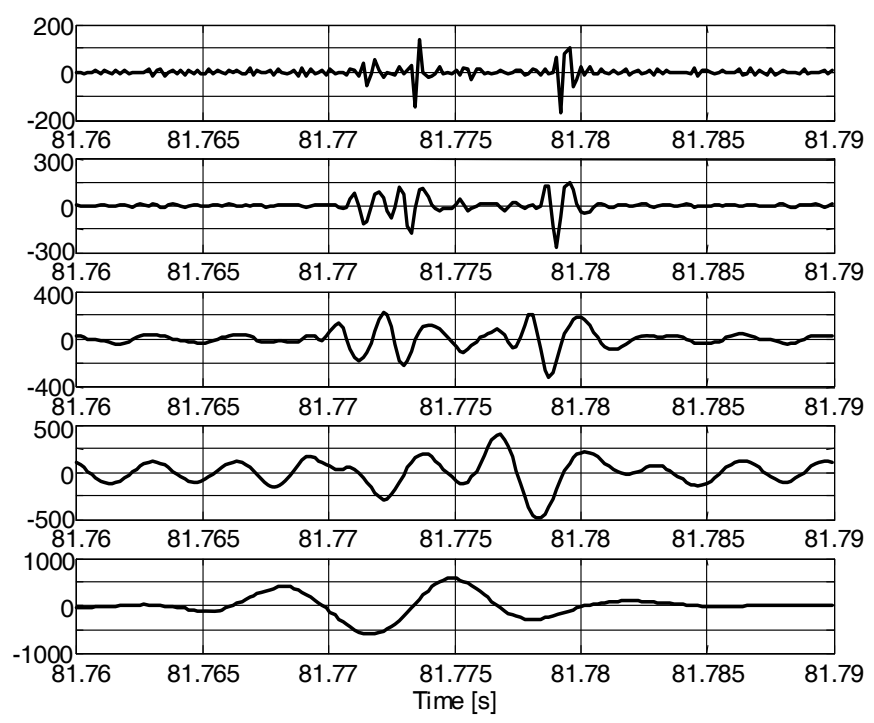

Figure 13. $V_{p}$ transient of Figure 8: db3, 5 levels.

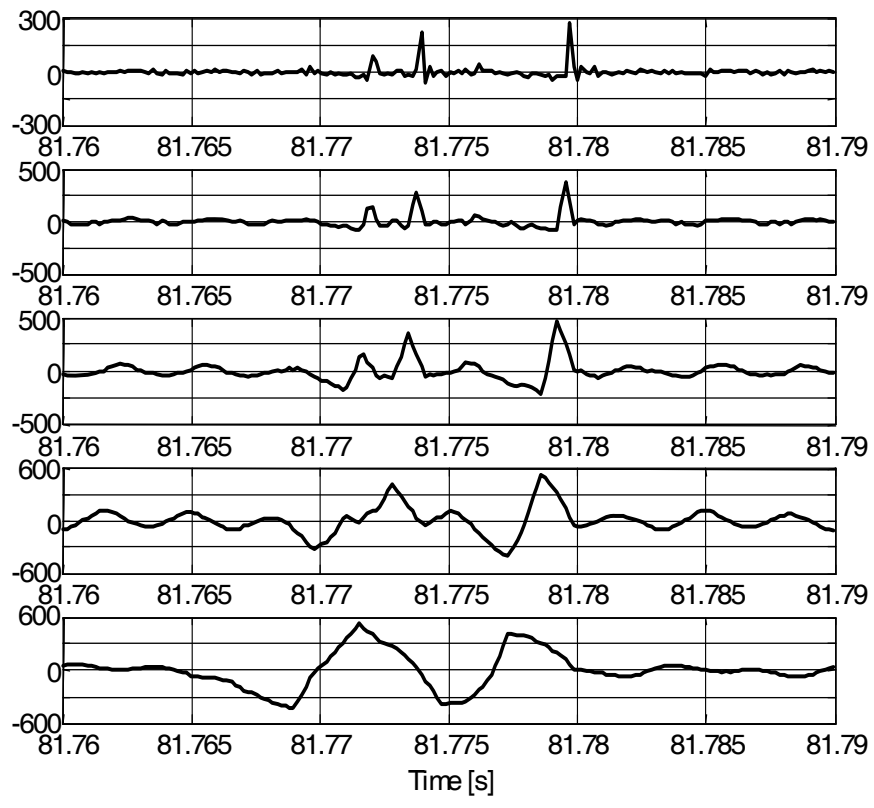

Figure 14. $V_{p}$ transient of Figure 8: db1, 5 levels (identical results for sym1 and Haar wavelets).

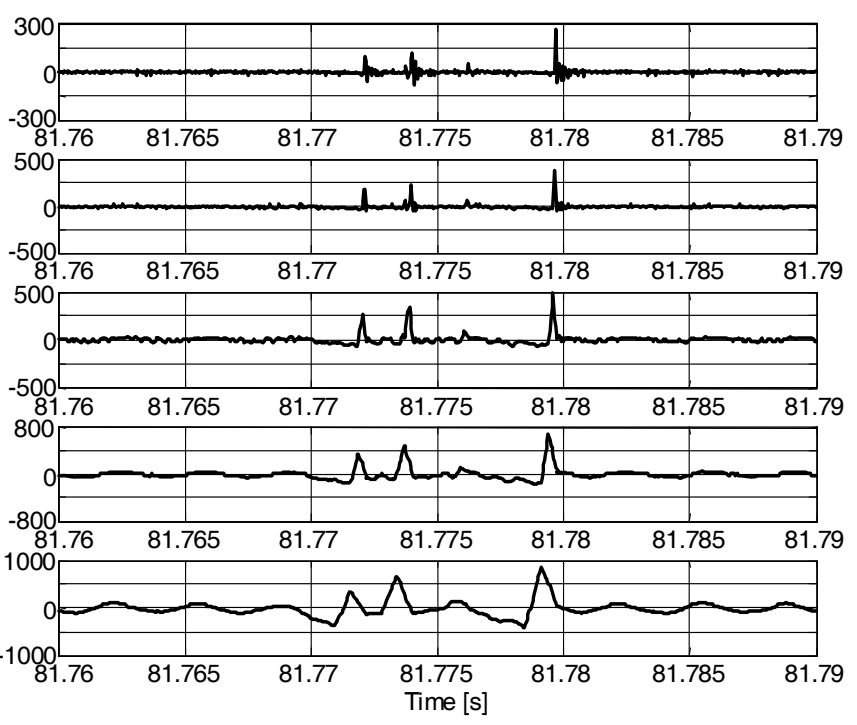

Figure 15. $V_{p}$ transient of Figure 8: db1, 5 levels ( $40 \mu$ s sample time).

\section{REFERENCES}

[1] A. Mariscotti, Direct Measurement of Power Quality over railway networks with results of a $16.7 \mathrm{~Hz}$ network, IEEE Transactions on Instrumentation and Measurement, 60 (2011), pp. 1604-1612.

[2] EN 50388, Railway applications - Power supply and rolling stock - Technical criteria for the coordination between power supply (substation) and rolling stock to achieve interoperability, Aug. 2005

[3] EN 61000-4-7, Electromagnetic compatibility - Part 4-7: Testing and measurement techniques - General guide on harmonics and interharmonics measurements and instrumentation, for power supply systems and equipment connected thereto, 2002-08.

[4] B. Hemmer, A. Mariscotti D. Wuergler, Recommendations for the calculation of the total disturbing return current from electric traction vehicles, IEEE Transactions on Power Delivery, 19 (2004), pp. 1190-1197.

[5] EN 50163, Railway applications - Supply voltages of traction systems, Nov. 2004.

[6] A. Mariscotti and D. Slepicka, "Analysis of frequency stability of 16.7 $\mathrm{Hz}$ railways", Proc. of the IEEE Intern. Instrumentation and Measurement Technical Conference I2MTC, May 10-12, 2011, Hangzhou, China.

[7] M. Meyer and G.J. van Alphen, Netzresonanzmessungen auf HSL Zuid und Betuweroute, Eisenbahn Review, 7-8 (2006), pp. 610-611.

[8] A. Dolara, M. Gualdoni and S. Leva, Impact of High Voltage Primary Supply Lines in the $2 \times 25 \mathrm{kV}-50 \mathrm{~Hz}$ Railway System on the Equivalent Impedance at Pantograph Terminals, IEEE Transactions on Power Delivery, 27 (2012), pp. 164-175.

[9] M. Meyer and J. Schöning, Netzstabilitat in Grossen Bahnnetzen, Eisenbahn Review, 7-8 (1999), pp. 312-317.

[10] prEN 50238-2 (draft, Pr. 15360), Railway applications Compatibility between Rolling Stock and Train Detection Systems - Part 2: Compatibility with Track Circuits, 2009.

[11] CLC/TR 50507, Railway applications - Interference limits of existing track circuits used on European railways, 2007-05.

[12] Specifica Ferrovie dello Stato (FS) N. 370582 esp. 1.0.

[13] A. Mariscotti, "DC railway line voltage ripple for periodic and aperiodic phenomena", Proc. of the IMEKO TC 04 Congress, Sept. 27-30, 2011, Natal, RN, Brazil.

[14] M. Caserza Magro, A. Mariscotti and P. Pinceti, "Definition of Power Quality Indices for DC Low Voltage Distribution Networks", IMTC 2006, Sorrento, Italy, April 20-23, 2006.

[15] EN 61000-4-17, Electromagnetic compatibility - Part 4-17: Testing and measurement techniques - Ripple on d.c. input power port immunity test, 1999-08. 
[16] MIL STD 704E, Aircraft electric power characteristics, May 1991.

[17] A. Mariscotti, Analysis of the dc link current spectrum in voltage source inverters, IEEE Transactions on Circuits and Systems Part I, 49 (2002), pp. 484-491.

[18] A. Mariscotti and P. Pozzobon, Synthesis of line impedance expressions for railway traction systems, IEEE Transactions on Vehicular Technology, 52 (2003), pp. 420-430.

[19] E.W. Kimbark, Direct Current Transmission, Wiley Interscience, 1971.

[20] A. Mariscotti, "Methods for ripple index evaluation in dc low voltage distribution networks", Proc. of the IEEE Instrumentation and Measurement Technical Conference IMTC, May 2-4, 2007, Warsaw, Poland.

[21] S. Herraiz Jaramillo, G.T. Heydt and Efrain O'Neill-Carrillo, Power Quality Indexes for Aperiodic Voltage and Currents, IEEE Transactions on Power Delivery, 15 (2000), pp. 784-790.

[22] A. Mariscotti, Discussion of Power Quality Indexes for Aperiodic Voltage and Currents, IEEE Transactions on Power Delivery, 15 (2000), pp. 1333-1334.

[23] CLC/TS 50238-2, Railway applications - Compatibility between rolling stock and train detection systems - Part 2: Compatibility with Track Circuits, 2010-07.
[24] A. Mariscotti, "On the uncertainty of the bandpass filter method for the evaluation of interference on track circuits", Proc. of the 20th IMEKO World Congress, Sept. 9-14, 2012, Busan, Republic of Korea.

[25] G. Armanino, A. Mariscotti and M. Mazzucchelli, "In-house test of low frequency conducted emissions of static converters for railway application", Proc. of the 17th IMEKO World Congress, June 22-27, 2003, Cavtat-Dubrovnik, Croatia.

[26] D. Bellan, A. Gaggelli, F. Maradei, A. Mariscotti and S. Pignari, Time-Domain Measurement and Spectral Analysis of NonStationary Low-Frequency Magnetic Field Emissions on Board of Rolling Stock, IEEE Transactions on Electromagnetic Compatibility, 46 (2004), pp. 12-23.

[27] L. Angrisani, P. Daponte, M. D'Apuzzo, and A. Testa, A measurement method based on the Wavelet Transform for power quality analysis, IEEE Transactions on Power Delivery, 13 (1998), pp. 990-998.

[28] A. Mariscotti and D. Slepicka, "The frequency stability of the $50 \mathrm{~Hz}$ French railway", Proc. of the IEEE International Instrumentation and Measurement Technical Conference, I2MTC, May 14-16, 2012, Graz, Austria. 